\title{
Multiplet-specific shape resonant features in photoionization of NO
}

\author{
Maile E. Smith ${ }^{\text {a) }}$ and Vincent McKoy

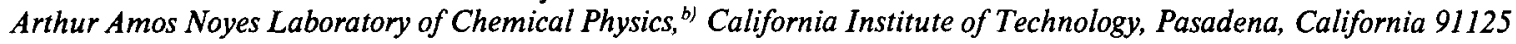

Robert R. Lucchese

Department of Chemistry, Texas A \& M University, College Station, Texas 77843

(Received 1 October 1984; accepted 15 October 1984)

\begin{abstract}
We report photoionization cross sections and asymmetry parameters for the $5 \sigma$ and $4 \sigma$ levels of NO obtained using numerical photoelectron continuum functions. For the $5 \sigma$ orbital multiplet-specific potentials lead to shape-resonant cross sections which show significant nonstatistical multiplet behavior. This behavior, which reflects the sensitivity of shape resonances to the exchange component of the molecular ion potential, is most prominently characterized by resonance peaks at substantially different photoelectron kinetic energies in the two multiplet channels. Comparison of the calculated cross sections with available experimental data for NO shows somewhat poorer agreement than has been seen in the photoionization of closed-shell systems such as $\mathrm{N}_{2}$ and $\mathrm{CO}$. This difference reflects the more complex nature of photoionization processes in open-shell molecules.
\end{abstract}

\section{INTRODUCTION}

Shape resonances in the $\sigma$ continuum are known to play an important role in the photoionization of many diatomic and linear polyatomic molecules. ${ }^{1-4}$ These resonances and their effects on the cross sections, angular distributions, and vibrational branching ratios have been extensively characterized by synchrotron radiation studies. ${ }^{1,4}$ Several approaches to the calculation of molecular photoionization cross sections have also been used to carry out related theoretical studies of these resonant processes. These approaches include the Stieltjes moment theory method, ${ }^{5}$ the continuum multiple-scattering model, ${ }^{6}$ and several methods for the direct solution of the Hartree-Fock equations for the photoelectron continuum orbitals. $^{7-12}$ Although the more general features of the resonant cross sections obtained by these different methods are quite similar for many systems, some physically important differences between these various results can be identified. For example, the muffin-tin approximation and local potential of the multiple-scattering model can lead to much larger vibrational averaging effects and deviations from Franck-Condon behavior than do staticexchange calculations. ${ }^{10.13 .14}$ In some cases Stieltjes moment theory studies have excessively broadened and even smoothed away pronounced resonance structure seen in calculations which directly use photoelectron continuum functions. ${ }^{10.13}$ These results illustrate the sensitivity of shape resonant photoionization cross sections to both the potentials and techniques used in their determination.

With some exceptions such as $\mathrm{O}_{2}{ }^{15}$ most systems studied to date have been closed-shell molecules. ${ }^{1.3,4}$ The

\footnotetext{
a) Permanent address: Institute for Defense Analyses, $1801 \mathrm{~N}$. Beauregard St., Alexandria, VA 22311.

b) Contribution No. 7094.
}

photoionization spectra of such molecules show no multiplet structure and the molecular ion potential is of a particularly simple form in the Hartree-Fock approximation. For this and other reasons the photoionization spectra of NO, an open-shell molecule with a $1 \sigma^{2} 2 \sigma^{2} 3 \sigma^{2} 4 \sigma^{2} 5 \sigma^{2} 1 \pi^{4} 2 \pi\left(X^{2} \Pi\right)$ ground electron configuration, should be particularly interesting. Ionization of the $2 \pi$ level leads to the $X^{1} \Sigma^{+}$closed-shell ground ionic state of $\mathrm{NO}^{+}$, whereas removal of the $5 \sigma$ and $4 \sigma$ electrons leads to the $b^{3} \Pi$ and $A^{1} \Pi$ terms and the $c^{3} \Pi$ and $B^{1} \Pi$ terms, respectively. Removal of the $1 \pi$ electron gives rise to six terms. ${ }^{16}$ Synchrotron radiation studies have determined the photoionization cross sections for production of the $X^{1} \Sigma^{+}, b^{3} \Pi, c^{3} \Pi,{ }^{17} c^{3} \Pi, B{ }^{1} \Pi,{ }^{18}$ and $b^{3} \Pi$, $A^{1} \Pi^{19}$ ions. These cross sections for photoionization of the $2 \pi, 5 \sigma$, and $4 \sigma$ orbitals exhibit the shape resonance feature expected in the $\sigma$ continuum. Moreover, in their recent measurements Morin et al. ${ }^{19}$ actually determined multiplet-specific cross sections for the $b^{3} \Pi$ and $A^{1} \Pi$ ions which show resonance peaks at quite different photoelectron kinetic energies. These differences in the photoionization cross sections for the two multiplets can be interpreted as arising from the different exchange potentials seen by the photoelectron in the two cases and clearly reflect the sensitivity of shape resonant cross sections to the choice of potential.

Related theoretical studies have shown that the $\sigma$ shape resonant feature in photoionization of the $2 \pi$ level is obscured by the large nonresonant $2 \pi \rightarrow k \pi$ and $k \delta$ contributions to this cross section. ${ }^{20-22}$ Vibrational averaging significantly dampened the resonant component in the multiple scattering cross sections for this level. ${ }^{20}$ Cross sections obtained with Hartree-Fock photoelectron continuum orbitals showed no indication of such pronounced vibrational effects. ${ }^{22}$ The overall agreement between these calculated cross sections and the experimental data, how- 
ever, is not very good and, in particular, the broad feature between photon energies of 20 and $40 \mathrm{eV}$ is not accounted for by these models. ${ }^{20-22}$ The Stieltjes-Tchebycheff cross sections for photoionization of the $5 \sigma$ and $4 \sigma$ level were obtained using a multiplet-averaged potential for the molecular ion core and also showed some structured features similar to those seen in the $2 \pi$ results and which are presumably artifacts of the calculation. ${ }^{10.22}$

In this paper we present photoionization cross sections for production of the $b^{3} \Pi, A^{1} \Pi$ and $c^{3} \Pi, B^{1} \Pi$ states of $\mathrm{NO}^{+}$arising from the ionization of the $5 \sigma$ and $4 \sigma$ levels of NO, respectively. In our studies we used Hartree-Fock photoelectron continuum orbitals. For the $5 \sigma$ level cross sections were obtained with both multipletspecific and multiplet-averaged molecular ion potentials but for the $4 \sigma$ level only the multiplet-averaged potential was used. The multiplet-specific and multiplet-averaged potentials led to significant changes in the $A{ }^{1} \Pi$ cross sections reflecting the sensitivity of this resonant cross section to the nonlocal component of the molecular ion potentials since these two potentials differ only in their exchange parts. These differences were much smaller for the $b^{3} \Pi$ cross sections as would be expected from the weighting of the states in the multiplet-averaged potential. Although the overall shapes of the calculated and measured photoionization cross sections are similar, there are some important differences. For example, the resonance peak in the calculated $b^{3} \Pi$ cross section occurred at somewhat higher energy than seen experimentally. This discrepancy was less pronounced for the $A{ }^{1} \Pi$ cross section.

An outline of the paper is as follows. In the next section we give a brief description of our method for obtaining the photoelectron continuum orbitals. In this section we also discuss the multiplet-specific static-exchange potentials used in these calculations. In the following section we discuss the results of our studies of the photoionization of the $5 \sigma$ and $4 \sigma$ levels of NO along with a comparison of these results with those of other methods and available experimental data.

\section{THEORETICAL DEVELOPMENTS}

\section{A. Solution of equations}

The rotationally unresolved, fixed-nuclei, photoionization cross section is given by ${ }^{2}$

$$
\sigma=\frac{4 \pi^{2} \omega}{3 c}\left|\left\langle\Psi_{i}(\mathbf{r} R)|; \mu| \Psi_{f}(\mathbf{r} ; R)\right\rangle\right|^{2},
$$

where $\mu$ is the dipole moment operator and $\omega$ the photon frequency. In Eq. (1) $\Psi_{i}$ represents the initial state of the molecule with $N$ bound electrons and $\Psi_{f}$ the final state with a photoelectron in the electronic continuum. For $\Psi_{i}$ we use the Hartree-Fock wave function. For $\Psi_{f}$ we use the frozen-core approximation in which the bound orbitals of the ion are constrained to be idential to those of $\Psi_{i}$ and the continuum orbital satisfies a one-electron Schrödinger equation of the form

$$
\left(\nabla^{2}-U_{\mathrm{dir}}-U_{\mathrm{ex}}+k^{2}\right) \phi_{\mathrm{k}}(\mathbf{r})=\sum_{i=1}^{n} \lambda_{i} \chi_{i},
$$

where the $\lambda_{i}$ are Lagrange multipliers which enforce orthogonality of $\phi_{\mathbf{k}}$ to occupied orbitals $\chi_{i}$ and $U_{\text {dir }}$ and $U_{\text {ex }}$ are twice the local and nonlocal components of the molecular ion potential. The potential of Eq. (2) is nonspherical and nonlocal. Several methods for solving such equations have recently been developed. ${ }^{7-13}$ In this work we solve Eq. (2) for the photoelectron continuum function $\phi_{k}$ by a method in which the direct potential $U_{\text {dir }}$ is treated exactly by numerical integration and the exchange or nonlocal component is approximated by a separable potential of the Schwinger form, ${ }^{12}$ i.e.,

$$
U_{\mathrm{ex}} \simeq \sum_{i, j}^{M} U_{\mathrm{ex}}\left|\alpha_{i}\right\rangle\left[U_{\mathrm{ex}}^{-1}\right]_{i j}\left\langle\alpha_{j}\right| U_{\mathrm{ex}},
$$

where the $\alpha_{i}$ are chosen to be Cartesian Gaussian functions. This approach to the solution of these equations for molecular electronic continuum orbitals is well suited for strongly polar molecular ions. The details of the method have been discussed previously ${ }^{12}$ and here we present only a very brief review. The solution of Eq. (2) can be written as

$$
\phi_{\mathbf{k}}=\phi_{\mathbf{k}}^{e}+\sum_{i} \lambda_{i} \phi^{x^{i}}
$$

where the functions $\phi_{\mathbf{k}}^{e}$ and $\phi^{x i}$ satisfy the equations

$$
\phi_{\mathbf{k}}^{e}=\phi_{\mathbf{k}}^{d}+G^{d} U_{\mathbf{e x}} \phi_{\mathbf{k}}^{e}
$$

and

$$
\phi^{x i}=G^{d} \chi_{i}+G^{d} U_{\mathrm{ex}} \phi^{x i} .
$$

In $\mathrm{Eq}$. (5a) $\phi_{\mathbf{k}}^{d}$ is the solution for the direct potential, i.e.,

$$
\left(\nabla^{2}-U_{\text {dir }}+k^{2}\right) \phi_{\mathrm{k}}^{d}=0
$$

and $G^{d}$ is the Green's function for this potential, i.e., $\left(\nabla^{2}\right.$ $\left.-U_{\mathrm{dir}}+k^{2} \pm i \epsilon\right)^{-1}$. In the solution of Eqs. (5) $U_{\mathrm{ex}}$ is approximated by the separable form of Eq. (3). The resulting equations along with Eq. (6) are then partialwave expanded and numerically integrated. For a given basis in Eq. (3) these solutions are approximate solutions of Eq. (2). They are, in fact, solutions of Eq. (2) but with $U_{\text {ex }}$ approximated as in Eq. (3). Although these solutions can be iteratively improved to yield the converged solutions of Eq. (2), ${ }^{12}$ we have found that it is generally not necessary to obtain such solutions and in these studies we will use the solutions of Eq. (2) with a suitable expansion basis in Eq. (3).

Finally, the Hartree-Fock photoionization cross sections provided by these photoelectron continuum orbitals can also be shown to be variationally stable. ${ }^{12}$

\section{B. Computational details}

We have used this procedure to study the photoionization cross sections and photoelectron angular distributions for the $5 \sigma$ and $4 \sigma$ orbitals of NO within the frozen-core approximation. The SCF wave function for NO was obtained with a $[5 s, 3 p, 1 d]$ basis of contracted Cartesian Gaussian functions. This same basis was used previously by Delaney et $a l^{21}$ in their moment theory studies of these photoionization cross sections. At the 
ground state equilibrium internuclear distance of 2.173 a.u. the SCF energy in this basis was -129.269 a.u.

The partial wave expansions used in the solution of Eqs. (5) and (6) were truncated at sufficiently high values so as to assure adequate convergence of the photoionization cross sections. For example, the maximum partial wave retained in the expansion of the scattering basis, i.e., the $\alpha$ 's of Eq. (3), was $l=24$. This was also the maximum partial wave retained in both the expansion of the scattering basis in the exchange matrix elements and in the expansion of $1 / r_{i j}$ in the direct potential. In the expansion of the nuclear potential we retained partial waves up to $l_{\max }=48$. In the expansion of the target orbitals in the exchange matrix elements the maximum partial wave was chosen so that the corresponding orbital normalization was better than 0.99 , e.g., $l_{i}^{\mathrm{ex}}=20$ and 10 for the $1 \sigma$ and $2 \pi$ orbitals, respectively.

The Gaussian basis used in the separable representation of the potential of Eq. (3) contains 14 functions all centered on the nuclei. This basis set is given in Table I. The radial integration grid extended out to 64 a.u. and contained 320 points. The radial integration step size ranged from 0.02 a.u. near the nuclei to 0.5 beyond $r$ $=14$ a.u.

\section{The scattering potentials}

Photoionization of the $5 \sigma$ orbital of NO produces $\mathrm{NO}^{+}$ions in both the $b^{3} \Pi$ and $A^{1} \Pi$ states with ionization potentials of 16.6 and $18.3 \mathrm{eV}$, respectively. The electronic configuration of these ions is [core] $5 \sigma 2 \pi$ where [core] represents the $1 \sigma^{2} 2 \sigma^{2} 3 \sigma^{2} 4 \sigma^{2} 1 \pi^{4}$ closed shells. The molecular ion potentials seen by the photoelectron are obviously different for these two final states of the ion and hence the photoelectron continuum orbitals are multiplet spe-
TABLE I. Basis sets used in the Schwinger separable potential.

\begin{tabular}{cll}
\hline \hline $\begin{array}{c}\text { Continuum } \\
\text { symmetry }\end{array}$ & $(l, m, n)^{\mathrm{a}}$ & \multicolumn{1}{c}{ Exponents $^{\mathrm{b}}$} \\
\hline \multirow{2}{*}{$\sigma$} & $(0,0,0)$ & $8.0,4.0,2.0,1.0,0.5$ \\
& $(0,0,1)$ & $1.0,0.25$ \\
$\pi$ & $(1,0,0)$ & $8.0,4.0,2.0,1.0,0.5$ \\
& $(1,0,1)$ & $1.0,0.25$ \\
\hline
\end{tabular}

a Cartesian Gaussian basis functions defined as

$\phi(\mathbf{r})=N\left(x-A_{x}\right)^{\prime}\left(y-A_{y}\right)^{m}\left(z-A_{z}\right)^{n} e^{-\alpha|\mathbf{r}-\mathbf{A}|^{2}}$.

${ }^{b}$ Functions are centered on both the nitrogen and oxygen.

cific. Since these multiplet-specific potentials differ essentially in their exchange parts, one does not normally expect a great sensitivity of the calculated photoionization cross sections to the choice of potential. Identical potentials have often been used for these different multiplet channels. This approximation, which has been made in previous studies of $5 \sigma$ photoionization of $\mathrm{NO}^{20,21}$ leads to cross sections that are exactly in the multiplet ratio as a function of kinetic energy, e.g., $3: 1$ in this case. Although multiplet-averaged potentials can be useful in nonresonant channels, important features such as the peak positions in shape-resonant cross sections may be poorly reproduced by such potentials. Photoionization of the $5 \sigma$ and $4 \sigma$ orbitals of NO provides an excellent example to illustrate these effects. In fact, we will see that these multipletspecific cross sections have resonance peaks at significantly different photoelectron kinetic energies, a feature which is completely masked by multiplet-averaged potentials.

The final state wave functions for photoionization of the $5 \sigma$ orbital leading to the $b^{3} \Pi$ state of $\mathrm{NO}^{+}$can be shown to be

$$
\begin{aligned}
\Psi\left({ }^{2} \Pi\right)= & \frac{1}{\sqrt{6}}\left\{2\left|[\operatorname{core}](5 \sigma)\left(2 \pi_{+}\right)(\overline{k \sigma})-\right|[\operatorname{core}](\overline{5 \sigma})\left(2 \pi_{+}\right)(k \sigma)|-|[\operatorname{core}](5 \sigma)\left(\overline{2 \pi_{+}}\right)(k \sigma) \mid\right\}, \\
\Psi\left({ }^{2} \Sigma^{+}\right)= & \frac{1}{\sqrt{12}}\left\{2\left|[\operatorname{core}](5 \sigma)\left(2 \pi_{+}\right)\left(\overline{k \pi_{-}}\right)\right|-\left|[\operatorname{core}](\overline{5 \sigma})\left(2 \pi_{+}\right)\left(k \pi_{-}\right)\right|-\left|[\operatorname{core}](5 \sigma)\left(\overline{2 \pi_{+}}\right)\left(k \pi_{-}\right)\right|\right. \\
& \left.+2\left|[\operatorname{core}](5 \sigma)\left(2 \pi_{-}\right)\left(\overline{k \pi_{+}}\right)\right|-\left|[\operatorname{core}](\overline{5 \sigma})\left(2 \pi_{-}\right)\left(k \pi_{+}\right)\right|-\left|[\operatorname{core}](5 \sigma)\left(\overline{2 \pi_{-}}\right)\left(k \pi_{+}\right)\right|\right\}, \\
\Psi\left({ }^{2} \Sigma^{-}\right)= & \frac{1}{\sqrt{12}}\left\{2\left|[\operatorname{core}](5 \sigma)\left(2 \pi_{+}\right)\left(\overline{k \pi_{-}}\right)\right|-\left|[\operatorname{core}](\overline{5 \sigma})\left(2 \pi_{+}\right)\left(k \pi_{-}\right)\right|-\left|[\operatorname{core}](5 \sigma)\left(\overline{2 \pi_{+}}\right)\left(k \pi_{-}\right)\right|\right. \\
& \left.-2\left|[\operatorname{core}](5 \sigma)\left(2 \pi_{-}\right)\left(\overline{k \pi_{+}}\right)\right|+\left|[\operatorname{core}](\overline{5 \sigma})\left(2 \pi_{-}\right)\left(k \pi_{+}\right)\right|+\left|[\operatorname{core}](5 \sigma)\left(\overline{2 \pi_{-}}\right)\left(k \pi_{+}\right)\right|\right\}, \\
\Psi\left({ }^{2} \Delta\right)= & \frac{1}{\sqrt{6}}\left\{2\left|[\operatorname{core}](5 \sigma)\left(2 \pi_{+}\right)\left(\overline{k \pi_{+}}\right)\right|-\left|[\operatorname{core}](\overline{5 \sigma})\left(2 \pi_{+}\right)\left(k \pi_{+}\right)\right|-\left|[\operatorname{core}](5 \sigma)\left(\overline{2 \pi_{+}}\right)\left(k \pi_{+}\right)\right|\right\} .
\end{aligned}
$$

With these wave functions the static-exchange one-particle Schrödinger equations satisfied by the continuum orbitals can be derived straightforwardly":

$$
\begin{aligned}
& { }^{2} \Pi: \quad P\left(f+\sum_{\text {core }}\left(2 J_{i}-K_{i}\right)+J_{5 \sigma}\right. \\
& \left.+J_{2 \pi}+\frac{1}{2} K_{5 \sigma}+\frac{1}{2} K_{2 \pi}-\epsilon\right) P|k \sigma\rangle=0,
\end{aligned}
$$

$$
\begin{aligned}
& { }^{2} \Sigma^{+}: \quad P\left(f+\sum_{\text {core }}\left(2 J_{i}-K_{i}\right)+J_{5 \sigma}+J_{2 \pi}\right. \\
& \left.+\frac{1}{2} K_{5 \sigma}+\frac{1}{2} K_{2 \pi}+S_{2 \pi}^{\prime \prime}+\frac{1}{2} S_{2 \pi}^{\prime}-\epsilon\right) P\left|k \pi_{+}\right\rangle=0, \\
& { }^{2} \Sigma^{-}: \quad P\left(f+\sum_{\text {core }}\left(2 J_{i}-K_{i}\right)+J_{5 \sigma}+J_{2 \pi}\right. \\
& \left.+\frac{1}{2} K_{5 \sigma}+\frac{1}{2} K_{2 \pi}-S_{2 \pi}^{\prime \prime}-\frac{1}{2} S_{2 \pi}^{\prime}-\epsilon\right) P\left|k \pi_{+}\right\rangle=0,
\end{aligned}
$$




$$
\begin{aligned}
& { }^{2} \Delta: \quad P\left(f+\sum_{\text {core }}\left(2 J_{i}-K_{i}\right)+J_{5 \sigma}\right. \\
& \left.+J_{2 \pi}+\frac{1}{2} K_{5 \sigma}+\frac{1}{2} K_{2 \pi}-\epsilon\right) P\left|k \pi_{+}\right\rangle=0 .
\end{aligned}
$$

In these equations $J_{i}$ and $K_{i}$ represent the usual Coulomb and exchange operators and $P$ is a projection operator which ensures the necessary orthogonality of the continuum orbitals to occupied orbitals. ${ }^{11}$ The energy of the photoelectron is given by $\epsilon$. The use of this projection operator is equivalent to the use of Lagrange multipliers in Eq. (2). The operators $S^{\prime \prime}$ and $S^{\prime}$ are defined by

$$
S_{\pi}^{\prime \prime} \phi_{+}\left(\mathbf{r}_{1}\right)=\phi_{-}\left(\mathbf{r}_{1}\right) \int d^{3} r_{2}\left[\pi_{-}\left(\mathbf{r}_{2}\right)\right]^{*} \frac{1}{r_{12}} \pi_{+}\left(\mathbf{r}_{2}\right)
$$

and

$$
S_{\pi}^{\prime} \phi_{+}\left(\mathbf{r}_{1}\right)=\pi_{+}\left(\mathbf{r}_{1}\right) \int d^{3} r_{2}\left[\pi_{-}\left(\mathbf{r}_{2}\right)\right]^{*} \frac{1}{r_{12}} \phi_{-}\left(\mathbf{r}_{2}\right)
$$

and

$$
f_{i}=-\frac{1}{2} \nabla_{i}^{2}=\sum_{\alpha} \frac{Z_{\alpha}}{r_{i \alpha}},
$$

where $Z_{\alpha}$ is the nuclear charge.

The final-state wave functions for photoionization of the $5 \sigma$ orbital leading to the $A^{1} \Pi$ ion are likewise

$$
\begin{aligned}
& \Psi\left({ }^{2} \Pi\right)= \frac{1}{\sqrt{2}}\left\{\left|[\operatorname{core}](5 \sigma)\left(\overline{2 \pi_{+}}\right)(k \sigma)\right|\right. \\
&\left.-\left|[\operatorname{core}](\overline{5 \sigma})\left(2 \pi_{+}\right)(k \sigma)\right|\right\}, \\
& \Psi\left({ }^{2} \Sigma^{+}\right)= \frac{1}{2}\left\{\left|[\operatorname{core}](5 \sigma)\left(\overline{2 \pi_{+}}\right)\left(k \pi_{-}\right)\right|\right. \\
&-\left|[\operatorname{core}](\overline{5 \sigma})\left(2 \pi_{+}\right)\left(k \pi_{-}\right)\right|+\left|[\operatorname{core}](5 \sigma)\left(\overline{2 \pi_{-}}\right)\left(k \pi_{+}\right)\right| \\
&\left.-\left|[\operatorname{core}](\overline{5 \sigma})\left(2 \pi_{-}\right)\left(k \pi_{+}\right)\right|\right\}, \\
& \Psi\left({ }^{2} \Sigma^{-}\right)=\frac{1}{2}\left\{\left|[\operatorname{core}](5 \sigma)\left(\overline{2 \pi_{+}}\right)\left(k \pi_{-}\right)\right|\right. \\
&-\left|[\operatorname{core}](\overline{5 \sigma})\left(2 \pi_{+}\right)\left(k \pi_{-}\right)\right|-\left|[\operatorname{core}](5 \sigma)\left(\overline{2 \pi_{-}}\right)\left(k \pi_{+}\right)\right| \\
&\left.+\left|[\operatorname{core}](\overline{5 \sigma})\left(2 \pi_{-}\right)\left(k \pi_{+}\right)\right|\right\}, \\
& \Psi\left({ }^{2} \Delta\right)=\frac{1}{\sqrt{2}}\left\{\left|[\operatorname{core}](5 \sigma)\left(\overline{2 \pi_{+}}\right)\left(k \pi_{+}\right)\right|\right. \\
&\left.\quad-\left|[\operatorname{core}](\overline{5 \sigma})\left(2 \pi_{+}\right)\left(k \pi_{+}\right)\right|\right\},
\end{aligned}
$$

and the related single-particle Schrödinger equations for the continuum orbitals are

$$
\begin{aligned}
& { }^{2} \Pi: \quad P\left[f+\sum_{\text {core }}\left(2 J_{i}-K_{i}\right)+J_{5 \sigma}\right. \\
& \left.+J_{2 \pi}-\frac{1}{2} K_{5 \sigma}-\frac{1}{2} K_{2 \pi}-\epsilon\right] P|k \sigma\rangle=0 \\
& { }^{2} \Sigma^{+}: \quad P\left[f+\sum_{\text {core }}\left(2 J_{i}-K_{i}\right)+J_{5 \sigma}+J_{2 \pi}-\frac{1}{2} K_{5 \sigma}\right. \\
& \left.-\frac{1}{2} K_{2 \pi}+S_{2 \pi}^{\prime \prime}-\frac{1}{2} S_{2 \pi}^{\prime}-\epsilon\right] P\left|k \pi_{+}\right\rangle=0,
\end{aligned}
$$

$$
\begin{aligned}
& { }^{2} \Sigma^{-}: \quad P\left[f+\sum_{\text {core }}\left(2 J_{i}-K_{i}\right)+J_{5 \sigma}+J_{2 \pi}-\frac{1}{2} K_{5 \sigma}\right. \\
& \left.-\frac{1}{2} K_{2 \pi}-S_{2 \pi}^{\prime \prime}+\frac{1}{2} S_{2 \pi}^{\prime}-\epsilon\right] P\left|k \pi_{+}\right\rangle=0 \\
& { }^{2} \Delta: \quad P\left[f+\sum_{\text {core }}\left(2 J_{i}-K_{i}\right)+J_{5 \sigma}\right. \\
& \left.+J_{2 \pi}-\frac{1}{2} K_{5 \sigma}-\frac{1}{2} K_{2 \pi}-\epsilon\right] P\left|k \pi_{+}\right\rangle=0 .
\end{aligned}
$$

The molecular ion potentials of Eqs. (8) and (13) which are appropriate for the $b^{3} \Pi$ and $A^{1} \Pi$ ions of $\mathrm{NO}^{+}$ differ essentially in their exchange components. Due to the sensitivity of resonant photoionization cross sections to nonlocal potentials, these differences are sufficient to lead to significant nonstatistical behavior of these multiplet-specific cross sections.

To obtain a multiplet-averaged potential we start with a final state wave function which is a linear combination of the wave functions of Eqs. (7) and (12) with a $3: 1$ weighting, respectively. This combination of wave functions results in a new set of wave functions in which the $5 \sigma$ electron is singlet coupled to the continuum electron. The wave functions of Eqs. (7) and (12) are the appropriate final state wave functions for ionization leading to the $b^{3} \Pi$ and $A{ }^{1} \Pi$ ions. With this choice of wave function, the resulting single-particle equations for the continuum orbitals can be shown to be ${ }^{11}$

$$
\begin{aligned}
& { }^{2} \text { II: } P\left[f+\sum_{\text {core }}\left(2 J_{i}-K_{i}\right)+J_{5 \sigma}\right. \\
& \left.+J_{2 \pi}+K_{5 \sigma}-\frac{1}{2} K_{2 \pi}-\epsilon\right] P|k \sigma\rangle=0 \\
& { }^{2} \Sigma^{+}: \quad P\left[f+\sum_{\text {core }}\left(2 J_{i}-K_{i}\right)+J_{5 \sigma}+J_{2 \pi}+K_{5 \sigma}\right. \\
& \left.-\frac{1}{2} K_{2 \pi}+S_{2 \pi}^{\prime \prime}-\frac{1}{2} S_{2 \pi}^{\prime}-\epsilon\right] P\left|k \pi_{+}\right\rangle=0 \\
& { }^{2} \Sigma^{-}: P\left[f+\sum_{\text {core }}\left(2 J_{i}-K_{i}\right)+J_{5 \sigma}+J_{2 \pi}+K_{5 \sigma}\right. \\
& \left.-\frac{1}{2} K_{2 \pi}-S_{2 \pi}^{\prime \prime}+\frac{1}{2} S_{2 \pi}^{\prime}-\epsilon\right] P\left|k \pi_{+}\right\rangle=0 \\
& { }^{2} \Delta: \quad P\left[f+\sum_{\text {core }}\left(2 J_{i}-K_{i}\right)+J_{5 \sigma}+J_{2 \pi}+K_{5 \sigma}\right. \\
& \left.-\frac{1}{2} K_{2 \pi}-\epsilon\right] P \mid k \pi+\pi_{+}=0 .
\end{aligned}
$$

The photoionization cross sections obtained with the continuum solution of these equations will be referred to as the multiplet-averaged cross sections.

These photoelectron continuum orbitals are then used straightforwardly to evaluate the dipole length and velocity matrix elements which are needed to obtain the photoionization cross sections and angular distributions. ${ }^{11}$ The ${ }^{2} \Sigma^{+}$and ${ }^{2} \Sigma^{-}$wave functions of Eqs. (7), however, do 
not define final states in which the photoelectron has a well-defined $m_{l}$ projection on the molecular axis. To obtain the asymmetry parameters we hence work with linear combinations of these states in which the $m_{l}$ of the photoelectron is \pm 1 .

\section{RESULTS AND DISCUSSION}

\section{A. Ionization of the $5 \sigma$ level}

In Fig. 1 we show the calculated photoionization cross sections leading to both the $b^{3} \Pi$ and $A^{1} \Pi$ ions. The photon energy scale assumes ionization potentials of 16.6 and $18.3 \mathrm{eV}$, respectively. ${ }^{16}$ The pronounced resonance feature in these cross sections is due to the wellknown shape resonance in the $\sigma$ continuum. ${ }^{17-21}$ Before comparing with available experimental data, we note that these results indicate significant nonstatistical multiplet behavior of these cross sections. Specifically, the resonance maxima for the $b^{3} I I$ and $A^{1} \Pi$ ions occur at photoelectron kinetic energies of about 8.8 and $5.2 \mathrm{eV}$, respectively. This behavior, which arises from differences in the exchange component of the appropriate molecular ion potentials, is obviously not seen in studies using a multiplet-averaged potential. ${ }^{20.21}$ Figure 2 shows the $b^{3} \Pi$ and $A^{1}$ II photoionization cross sections obtained with the multiplet-averaged and multiplet-specific potentials. As expected, the differences between these cross sections are much larger for the $A{ }^{1} \Pi$ state than for the $b^{3} \Pi$ state. The multiplet-averaged static-exchange $5 \sigma$ photoionization cross sections of Delaney et al. ${ }^{21}$ obtained with the Stieltjes moment theory method showed irregular structure which is presumably spurious and due to the imaging procedure used. ${ }^{21}$ This spurious structure was also seen in the cross sections for the $2 \pi$ level..$^{21,22}$

In Fig. 3 we compare our calculated cross sections, both length and velocity forms, for the $b^{3} \Pi$ state with the experimental data of Southworth et al. ${ }^{17}$ These measured cross sections ${ }^{17}$ were obtained using synchrotron radiation. The calculated cross sections show a resonance maximum about $2 \mathrm{eV}$ higher than is seen experimentally.

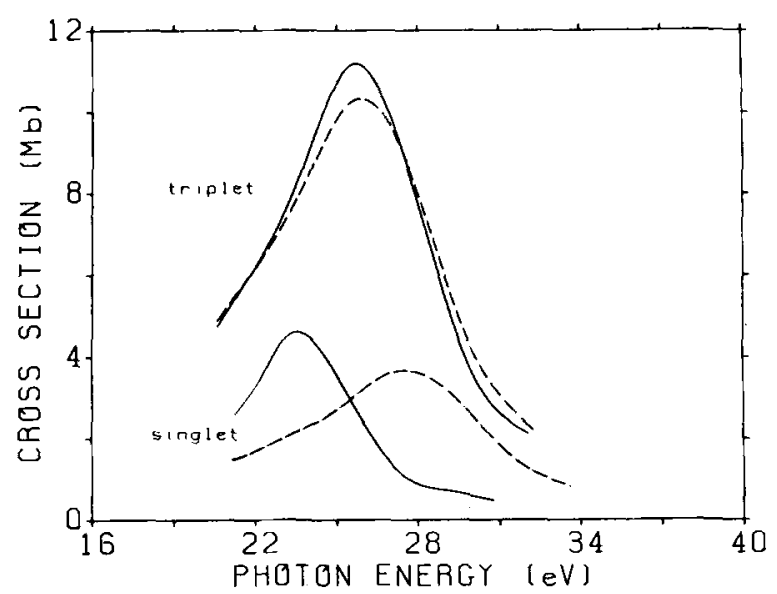

FIG. 1. Multiplet-specific cross sections for the $b^{3} \Pi$ and $A{ }^{1} I I$ states of $\mathrm{NO}^{+}:(-)$present results in the dipole-length form; $(--)$present results in the dipole-velocity form.

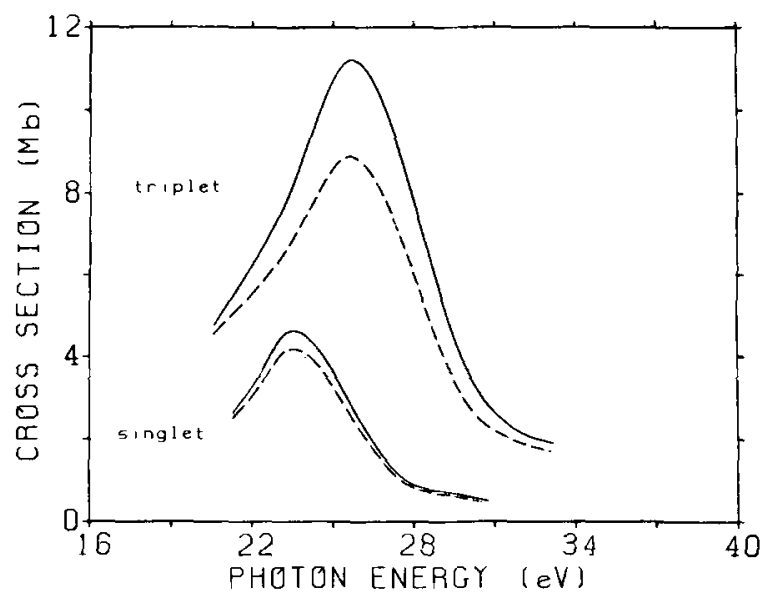

FIG. 2. Dipole-length cross sections for the $b^{3} \Pi$ and $A$ ' $\Pi$ states of $\mathrm{NO}^{+}:(-)$present results with a multiplet-specific potential; (- $)$present results with a multiplet-averaged potential.

Moreover, the peak height of our resonance is larger and its width is narrower than observed. We note, however, that there is a significant difference between the peak values of the length and velocity forms of this resonant cross section. This difference again emphasizes the importance of electron correlation effects on resonant cross sections. ${ }^{11,23}$ Vibrational averaging will tend to reduce and broaden these cross sections ${ }^{24}$ but the agreement between these fixed-nuclei, static-exchange photoionization cross sections and the experimental data is obviously poorer for NO than for related levels in $\mathrm{N}_{2}{ }^{11}$ and $\mathrm{CO}^{23}$ These differences reflect the more complex open-shell structure of $\mathrm{NO}^{+}$. In Fig. 3 we also show the fixed-nuclei cross sections obtained with the continuum multiple-scattering model. ${ }^{20}$ This model gives a resonance width which is narrower than is seen in our static-exchange results but the resonance positions are about the same. Vibrational averaging leads to significant, ${ }^{20}$ but probably excessive, ${ }^{13}$ broadening of these fixed-nuclei multiple-scattering cross sections.

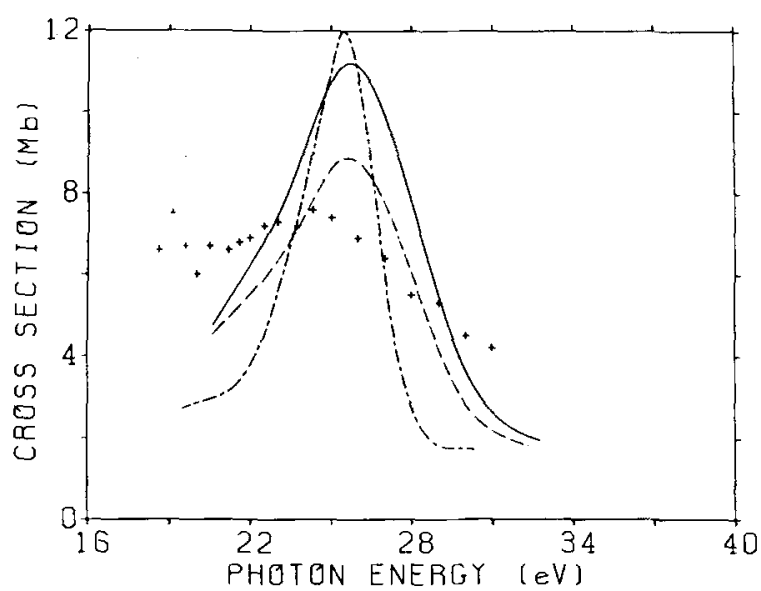

FIG. 3. Photoionization cross sections for the $b^{3} \Pi$ state of $\mathrm{NO}^{+}:(-)$ present results for a multiplet-specific potential in the dipole-length approximation; (- ) present results for a multiplet-specific potential in the dipole-velocity approximation; (- - ) multiplet-averaged potential in the CMSM of Ref. 20; $(+)$ experimental results of Ref. 17 . 
The vibrationally resolved $(v=0,1)$ photoionization cross sections for the $b^{3} \Pi$ and $A{ }^{1} \Pi$ ions are also being studied by Morin et al. ${ }^{19}$ Although preliminary unnormalized results have been published for these cross sections, it seems advisable not to reproduce these results in our figures at this time. ${ }^{24}$ Some comments, however, are in order. Although the observed resonance positions are lower than in our calculated cross sections, the resonance feature does occur at about $3 \mathrm{eV}$ higher in kinetic energy in the $b^{3} \Pi$ than in the $A{ }^{1} \Pi$ cross sections. The underlying resonance structure in these $b^{3} \Pi$ cross sections also appears narrower than in the data of Southworth $e t$ al. ${ }^{17}$ Moreover, the unnormalized cross sections for the $v=0$ and 1 levels of the $b^{3} \Pi$ channel indicate substantial non-Franck-Condon behavior. ${ }^{19}$

Figure 4 shows our calculated photoelectron asymmetry parameters for the $b^{3} \Pi$ state along with those of the multiple-scattering mode ${ }^{20}$ and the measured values of Southworth et al. ${ }^{17}$ The structure in these parameters between 17 and $22 \mathrm{eV}$ is due to autoionization which is not included in our model. Although the agreement between our calculated values and the experimental measurements is reasonable, it is again poor compared with the behavior seen in photoionization of related levels of $\mathrm{N}_{2}{ }^{11}$ and $\mathrm{CO}^{23}$

\section{B. Ionization of the $4 \sigma$ level}

Photoionization of the $4 \sigma$ level leads to the $c^{3} \Pi$ and $B{ }^{2} \Pi$ states. Unfortunately the assignment of these states in the photoelectron spectra is still controversial. One assignment associates both the $c^{3} \Pi$ and $B^{1} \Pi$ states with the band at $21.7 \mathrm{eV} .{ }^{16} \mathrm{An}$ alternative assignment puts the $c^{3} \Pi$ state at $21.7 \mathrm{eV}$ and suggests that the $B{ }^{1} \Pi$ state is strongly modified by configuration interaction and shifted to $22.7 \mathrm{eV} .{ }^{25}$ In view of these uncertainties and significant discrepancies between experimental data, we will obtain the photoionization cross sections for these channels using only the multiplet-averaged potential.

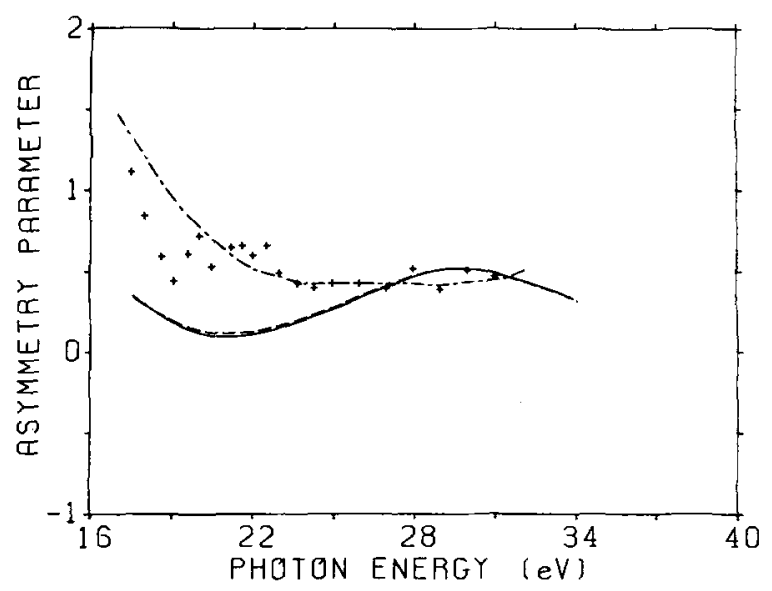

FIG. 4. Asymmetry parameters for the $b^{3} \Pi$ state of $\mathrm{NO}^{+}:(-)$present results for a multiplet-averaged potential in the dipole-length approximation; (-) present results for a multiplet-averaged potential in the dipole-velocity approximation; (- - ) multiplet-averaged potential in the CMSM of Ref. 20; $(+)$ experimental results of Ref. 17.

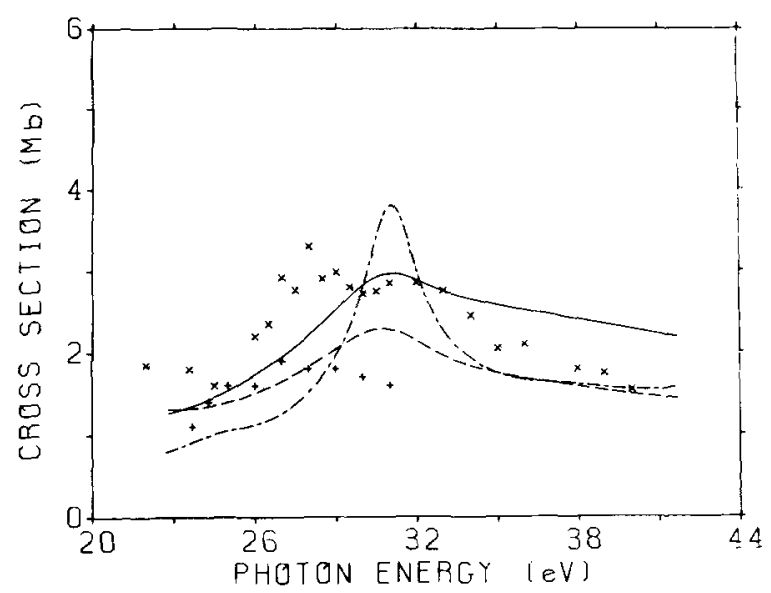

FIG. 5. Photoionization cross sections for the $c{ }^{3} \Pi$ state of $\mathrm{NO}^{+}:(-)$ present results for a multiplet-averaged potential in the dipole-length approximation; (- - ) present results for a multiplet-averaged potential in the dipole-velocity approximation; (- - ) multiplet-averaged potential in the CMSM of Ref. $20 ;(+)$ experimental results of Ref. $17 ;(X)$ experimental results of Ref. 18.

In Fig. 5 we show our calculated cross sections for the $c^{3} \Pi$ state along with the multiple scattering results ${ }^{20}$ and the experimental data of Southworth et al. ${ }^{17}$ and Gustaffson and Levinson. ${ }^{18}$ The photon energy scale assumes an ionization potential of $21.7 \mathrm{eV}$. The $\sigma$ shape resonance feature is again evident in these cross sections. Our calculated cross sections are in better agreement with the synchrotron radiation data of Gustaffson. ${ }^{18}$ There is also a significant difference between the length and velocity forms of these cross sections. However, the synchrotron radiation data of Southworth et al., ${ }^{17}$ which is substantially lower than that of Ref. 18 , is apparently in better agreement with the $(e, 2 e)$ data. ${ }^{17,26}$ Some further experimental studies of these cross sections would be very helpful. Figure 6 shows our calculated asymmetry parameters, those of the multiple-scattering model, and the experimental data of Southworth et al. ${ }^{17}$ The agreement between

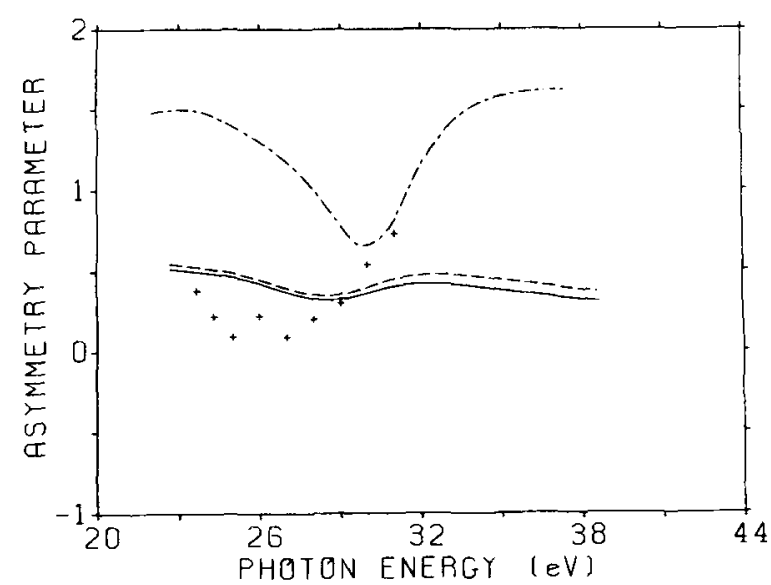

FIG. 6. Asymmetry parameters for the $c^{3} \Pi$ state of $\mathrm{NO}^{+}:(-)$present results for a multiplet-averaged potential in the dipole length approximation; (-) present results for a multiplet-averaged potential in the dipole-velocity approximation (- - ) multiplet-averaged potential in the CMSM of Ref. $20 ;(+)$ experimental results of Ref. 17. 


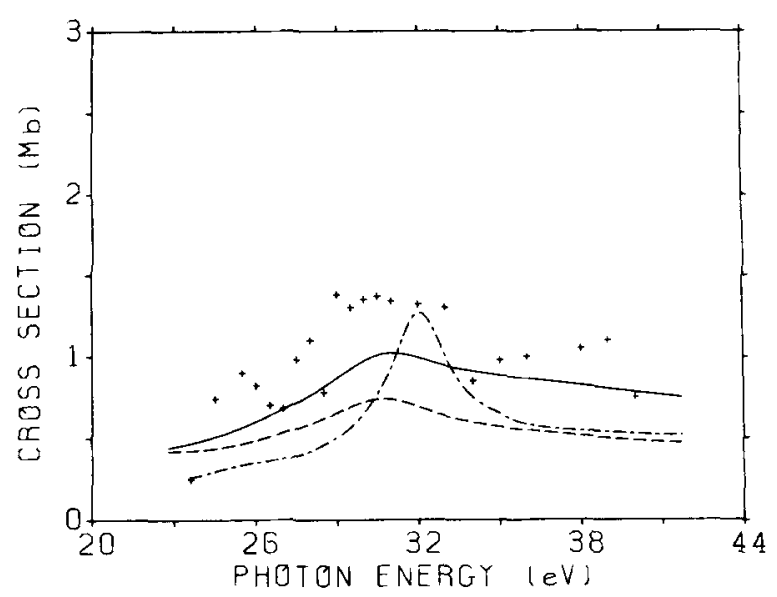

FIG. 7. Photoionization cross sections for the $B^{\prime} \Pi$ state of $\mathrm{NO}^{+}:(-)$ present results for a multiplet averaged potential in the dipole-length approximation; (- -) present results for a multiplet-averaged potential in the dipole-velocity approximation; (-. - multiplet-averaged potential in the CMSM of Ref. 20; $(+)$ experimental results of Ref. 18.

our calculated results and the measured asymmetry parameters is fair. The calculated asymmetry parameters for this level are expectedly quite similar to those of the $5 \sigma$ level. The measured values for this level show a deep minimum which is not seen in the data for the $5 \sigma$ level.

Figure 7 shows our calculated cross sections for the $B{ }^{1} \Pi$ level. In this figure we have assumed an ionization potential of $22.7 \mathrm{eV}$. Since we have used a multipletaveraged potential the cross sections for this state and those of the $c{ }^{3} \Pi$ state are in a $1: 3$ ratio. Again the agreement between the calculated and measured photoionization cross sections is quite good.

\section{CONCLUSION}

We have studied the cross sections for photoionization of the $5 \sigma$ and $4 \sigma$ levels of NO. These cross sections for the $b^{3} \Pi\left(5 \sigma^{-1}\right)$ and $A{ }^{1} \Pi\left(5 \sigma^{-1}\right)$ states show nonstatistical multiplet-specific effects which arise from the sensitivity of the $\sigma$ shape resonance in these channels to the exchange component of the potential seen by the photoelectron. Specifically, the resonance peak in the $A^{1} \Pi$ cross section appears at a kinetic energy about $3 \mathrm{eV}$ lower than in the $b^{3} \Pi$. This result certainly emphasizes the importance of using adequate potentials in studies of shape resonance features in molecular photoionization. This multipletspecific effect has also been observed experimentally. ${ }^{19}$ However, in these experiments the resonances were observed at energies lower than predicted here. The calculated peak heights of these resonance cross sections are somewhat larger than the data of Southworth et al. ${ }^{17}$ which, however, appears smaller than the preliminary data of Morin et al. ${ }^{19}$ The agreement here for these resonant cross sections in NO is certainly not as good as we have seen in related processes in $\mathrm{N}_{2}{ }^{11}$ and $\mathrm{CO} .{ }^{23}$ These differences are due in part to the complex open-shell structures of NO and suggest that studies beyond the Hartree-Fock approximation are needed. We note that in photoioniza- tion of the $2 \pi$ level leading to the closed-shell ${ }^{1} \Sigma^{+} \mathrm{NO}^{+}$ ion, substantial discrepancies between the calculated and observed cross sections were also seen. ${ }^{22}$

After the completion of this paper the results of Stieltjes moment theory studies of the photoionization cross sections for the $b^{3} \Pi$ and $A^{1} \Pi\left(5 \sigma^{-1}\right)$ states have been published. ${ }^{27}$ These results also show multiplet-specific behavior for these cross sections very similar to what we have seen. However, although the same model is used in these two studies, the resonance features in the Stieltjes moment theory calculations are significantly broader than in our cross sections of Fig. 1. Moreover, the resonance position is at somewhat lower energy. These sorts of differences between photoionization cross sections obtained with numerical single-center photoelectron continuum orbitals and from Stieltjes moment theory studies have been seen previously in many systems. ${ }^{10,28,29}$ The underlying reasons for this behavior have been discussed extensively elsewhere. ${ }^{2,4,10,30}$

\section{ACKNOWLEDGMENTS}

One of us (MES) acknowledges support of a National Science Foundation predoctoral fellowship and also thanks Dr. Marvin Goodgame for several helpful conversations. This material is based upon research supported by the National Science Foundation under Grant No. CHE8218166. The authors acknowledge computing support from the National Center for Atmospheric Research (NCAR) which is sponsored by the National Science Foundation. The research reported in this paper made use of the Dreyfus-NSF Theoretical Chemistry Computer which was funded through grants from the Camille \& Henry Dreyfus Foundation, the National Science Foundation (Grant No. CHE-7820235), and the Sloan Fund of the California Institute of Technology.

${ }^{1}$ J. L. Dehmer, D. Dill, and A. C. Parr, in Photophysics and Photochemistry in the Vacuum Ultraviolet, edited by S. McGlynn, G. Findley, and R. Huebner (Reidel, Holland, 1984).

${ }^{2}$ See, for example, D. Lynch, V. McKoy, and R. R. Lucchese, in Symposium on Resonances in Electron-Molecule Scattering, van der Waals Complexes, and Reactive Chemical Dynamics, edited by D. G. Truhlar, ACS Symp. Ser. 263 (American Chemical Society, Washington, D.C., 1984).

${ }^{3}$ P. W. Langhoff, N. Padial, G. Csanak, T. N. Rescigno, and B. V. McKoy, J. Chem. Phys. 77, 590 (1980).

${ }^{4}$ See, for example, V. McKoy, T. A. Carlson, and R. R. Lucchese, J. Phys. Chem. 88, 3188 (1984).

${ }^{5}$ P. W. Langhoff, in Electron-Molecule and Photon-Molecule Collisions, edited by T. N. Rescigno, B. V. McKoy, and B. Schneider (Plenum, New York, 1979), pp. 183-224.

${ }^{6}$ D. Dill and J. L. Dehmer, J. Chem. Phys. 61, 692 (1974).

${ }^{7}$ G. Raseev, H. Le Rouzo, and H. Lefebvre-Brion, J. Chem. Phys. 72, 5701 (1980).

${ }^{8}$ W. D. Robb and L. A. Collins, Phys. Rev. A 22, 2474 (1980).

${ }^{9}$ T. N. Rescigno and A. E. Orel, Phys. Rev. A 24, 1267 (1982).

${ }^{10}$ B. I. Schneider and L. A. Collins, Phys. Rev. A 29, 1695 (1984).

"R. R. Lucchese, G. Raseev, and V. McKoy, Phys. Rev. A 25, 2572 (1982).

${ }^{12}$ M. E. Smith, V. McKoy, and R. R. Lucchese, Phys. Rev. A 29, 1857 (1984).

${ }^{13}$ R. R. Lucchese and V. McKoy, Phys. Rev. A 26, 1992 (1982). 
${ }_{14}^{14}$ R. R. Lucchese and V. McKoy, J. Phys. B 14, L629 (1981).

${ }^{15}$ See, for example, P. Morin, I. Nenner, M. Y. Adam, M. J. HubinFranskin, J. Delwiche, H. Lefebvre-Brion, and A. Giusti-Suzor, Chem. Phys. Lett. 92, 609 (1982). See also A. Gerwer, C. Asaro, B. V. McKoy, and P. W. Langhoff, J. Chem. Phys. 72, 713 (1980). No multiplet-specific potentials were used in these studies of Gerwer $e t$ al.

${ }^{16}$ O. Edgvist, L. Åsbrink, and E. Lindholm, Z. Naturforsch. Teil A 26, 1407 (1971).

${ }^{17}$ S. Southworth, C. M. Truesdale, P. H. Kobrin, D. W. Lindle, W. D. Brewer, and D. A. Shirley, J. Chem. Phys. 76, 143 (1982).

${ }^{18}$ T. Gustafsson and H. J. Levinson, Chem. Phys. Lett. 78, 28 (1981).

${ }^{19}$ P. Morin, M. Y. Adam, P. Lablanquie, I. Nenner, M. J. HubinFranskin, and J. Delwiche, Vacuum Ultraviolet Radiation Physics, VUV VII, Proceedings of the 7th International Conference (Israel Physical Society, Jerusalem, 1983), p. 613.
${ }^{20}$ S. Wallace, D. Dill, and J. L. Dehmer, J. Chem. Phys. 76, 1217 (1982).

${ }^{21}$ J. J. Delaney, I. H. Hillier, and V. R. Saunders, J. Phys. B 15, 1477 (1982).

${ }^{22}$ M. E. Smith, R. R. Lucchese, and V. McKoy, J. Chem. Phys. 79, 1360 (1983).

${ }^{23}$ R. R. Lucchese and V. McKoy, Phys. Rev. A 28, 1382 (1983).

${ }^{24} \mathrm{M}$. Y. Adam (private communication).

${ }^{25} \mathrm{H}$. Lefebvre-Brion, Chem. Phys. Lett. 9, 463 (1971).

${ }^{26}$ C. E. Brion and K. H. Tan, J. Electron. Spectrosc. Relat. Phenom. 23, 1 (1981).

${ }^{27}$ M. R. Hermann, S. R. Langhoff, and P. W. Langhoff, Chem. Phys. Lett. 109, 150 (1984).

${ }^{28}$ R. R. Lucchese and V. McKoy, J. Phys. Chem. 85, 2166 (1981).

${ }^{29} \mathrm{~B}$. I. Schneider and L. Collins (private communication).

${ }^{30}$ D. L. Lynch and V. McKoy, Phys. Rev. A 30, 1561 (1984). 